\title{
THE EFFECT OF ULTRAVIOLET B IRRADIATION ON THE ISOLATED PORCINE STRATUM CORNEUM: COLORIMETRIC AND ATR/FT-IR SPECTROSCOPIC INVESTIGATIONS
}

\author{
SHAN-YANG LIN and RUN-CHU LIANG \\ Biopharmaceutics Laboratory, Department of Medical Research, Veterans General Hospital-Taipei, Taipei, Republic of China
}

\begin{abstract}
To investigate the effect of ultraviolet B (UVB) on the isolated stratum corneum (SC), attenuated total reflectance/Fourier transform infrared spectroscopy and tristimulus colorimeter were used to evaluate the changes in secondary structure of proteins and in skin color following UVB irradiation. After UVB radiation, the color of porcine SC became blanching with the increase of exposure time, because melanin pigmentation did not occur in the isolated porcine SC. The peaks assigned to lipid structure disappeared, and some peaks split into additional peaks and/or shifted to lower frequency, possibly because of the unsaturation of protein and lipid in the porcine SC after UVB irradiation. Moreover, the peak area of amide II was decreased with the exposure time, due to the denaturation of protein induced by UVB. The $\alpha$-helix structure was transformed into $\beta$-sheet structure after UVB irradiation on the isolated porcine SC.
\end{abstract}

The photoaging and photodamaging effects of solar irradiation are well-established $(1,22,25)$. Excessive exposure of the skin to sunlight causes erythema, melanization, skin cancer and tissue damage $(7,13,30)$. These extensive alterations result mainly from ultraviolet B (UVB) $(6,20)$. Histologically, there are a number of alterations in the epidermis and dermis after UV irradiation such as increased skin surface lipids, basophilic degeneration of collagen, accumulation of elastotic material into dermis and an increase in glycosaminoglycans $(5,15,28)$.

Many immunochemical and biochemical studies have been made to determine the changes in collagen, damage of DNA, changes in ultrastructural features, increase in the horny cell layers and surface lipids after UV irradiation $(15,26,28)$. The changes in biophysical properties of human skin after sun-exposure have also been investigated (1). The noninvasive bioengineering technique is used to analyze the changes in vascular reactions after UVB exposure by using computerized reflectance spectroscopy (3). Recently, Fourier transform infrared (FT-IR) spectroscopy has been used to obtain useful information on protein secondary structures in non-disrupted biological materials $(10,18)$. Several IR spectroscopic studies on skin have been made after treatment with heat or penetration enhancers $(14,21)$. Lin et al. studied the conformational structure of stratum corneum from different animals by treatment with or without enhancers $(16,17)$. Much less attention has been paid to the IR spectroscopic changes in the conformational structure of the photodamaged-skin.

This study was attempted to investigate changes in the secondary structure of the isolated porcine stratum corneum (SC) after UVB irradiation using FT-IR spectroscopy with attenuated total reflectance (ATR) prism. The colorimetric assessment of the UVB-induced porcine SC was also made.

\section{MATERIALS AND METHODS}

\section{Isolation of Porcine Stratum Corneum}

$\mathrm{SC}$ of pig was prepared from its excised skin by heat separation $\left(60^{\circ} \mathrm{C}, 1 \mathrm{~min}\right)$ and subsequent digestion in $0.5 \%$ trypsin for $3 \mathrm{~h}$, as described previously (16). 
Control of both time and temperature was essential in the isolation procedure as temperature at $65^{\circ} \mathrm{C}$ and heating for $2 \mathrm{~min}$ can result in denaturation of protein or in imcomplete separation of the epidermis (12). The porcine SC was then isolated and airdried on a filter paper at room temperature and stored at desiccator until use. The isolated SC does not seem to degenerate, because its differential scanning calorimetric thermogram was comparable to other observation (9).

\section{UVB Irradiation}

The porcine SC was placed in a cabinet (Model CC10, chromato-UVE, UVP, U.S.A.) and exposed at a distance of $18 \mathrm{~cm}$ from a UV irradiation source that emits $350 \pm 25 \mathrm{uW} / \mathrm{cm}^{2}$ of UVB range with a peak at $302 \mathrm{~nm}$ (UVM-57, Chromato-UVE lamp, UVP, U.S.A.). The intensity of UVB irradiation was measured by a UVX radiometer (UVP, U.S.A.) with a sensor. At the described intervals, the SC irradiated was withdrawn and examined by tristimulus colorimetry and FT-IR spectroscopy.

\section{Porcine SC Color Measurements}

The porcine SC samples after UVB irradiation were examined by tristimulus colorimetry (Minolta Chroma Meter CR-200, Japan) in the $\mathrm{L}^{*} \mathrm{a}^{*} \mathrm{~b}^{*}$ color space. The reflective color of any surface can be quantified in a 3-dimensional coordinate system. The $\mathrm{L}^{*}$ value gives the relative brightness, ranging from black to white; the $\mathrm{a}^{*}$ value is the component of separation between red and green; the $b^{*}$ value represents the balance between yellow and blue. Calibration can be done by using a white plate. The total color difference is the magnitude of the resultant vector of three component differences, $\Delta \mathrm{L}^{*}$, $\Delta \mathrm{a}^{*}$ and $\Delta \mathrm{b}^{*}$. The total magnitude of color difference $(\Delta E)$ is a scalar quantity and may be computed from the following equation:

$$
\Delta \mathrm{E}=\left[\left(\Delta \mathrm{L}^{*}\right)^{2}+\left(\Delta \mathrm{a}^{*}\right)^{2}+\left(\Delta \mathrm{b}^{*}\right)^{2}\right]^{1 / 2}
$$

\section{ATR/FT-IR Spectroscopy}

IR spectra of the porcine SC samples after UVB irradiation were obtained using an FT-IR spectrophotometer equipped with an MCT detector and a zinc selenide ATR prism (Micro FT-IR 200, Jasco, Japan). The spectra were taken at $4 \mathrm{~cm}^{-1}$ resolution and generally 200 scans were accumulated to obtain a reasonable single-noise ratio.
The statistical analysis was performed based on the means of the triplicate data. Mean and standard deviation were obtained.

\section{RESULTS}

Changes in Porcine SC Color after UVB Exposure

Parameters $L^{*}, a^{*}$ and $b^{*}$ for the isolated porcine SC following UVB irradiation are illustrated as a function of exposure time (Fig. 1). A positive movement on the $L^{*}$-axis (toward white), a negative movement on the $a^{*}$-axis (toward green), and a positive movement on the $\mathrm{b}^{*}$-axis (toward yellow) were obtained. In addition, the value of the total color difference $(\Delta \mathrm{E})$ was approaching to zero, indicating the whitening of porcine SC after UVB irradiation. This result was in reverse to the usual erythematous response of the skin to solar exposure. Increasing darkening due to negative $L^{*}$ value and a marked increase in redness (positive a* value) were found in normal live skin (27).

\section{IR Spectra Shift of Porcine SC after UVB Irra- diation}

The secondary structure of protein can be studied from its IR spectrum by use of amide I and amide II frequencies as key indicators (11). A correlation between the secondary structure of proteins and amide I and amide II frequencies has been established not only for $\alpha$-helix, $\beta$-sheet and random coil structures but also for the triple helix structure (10, 11 ), but the amide I is much more sensitive than the amide II (4). Fig. 2 shows the results of the exposure time-dependent IR spectra. Apparently, the peak intensity of $1,743 \mathrm{~cm}^{-1}$ (assigned to the carbonyl group of lipid) and $1,466 \mathrm{~cm}^{-1}$ (assigned to the $\mathrm{CH}_{2}$ scissoring band of lipid) decreased with the exposure time. The IR spectra of amide I and II split into two or three peaks. The peak at $1,454 \mathrm{~cm}^{-1}$ assigned to $\mathrm{CH}_{3}$ asymmetric bending shifted to $1,446 \mathrm{~cm}^{-1}$ after UVB irradiation. By increasing the exposure time, the peak at 1,396 $\mathrm{cm}^{-1}$, assigned to $\mathrm{CH}_{2}$ and $\mathrm{CH}_{3}$ deformation and/ or $\mathrm{COO}^{-}$symmetric stretching band, split into two peaks at 1,412 and $1,383 \mathrm{~cm}^{-1}$ following 36 days of continuous irradiation. The peak at 1,057 $\mathrm{cm}^{-1}$, assigned to the C-O stretching coupled with $\mathrm{C}-\mathrm{O}$ bending of the $\mathrm{C}-\mathrm{OH}$ groups of carbohydrates, transferred to $1,043 \mathrm{~cm}^{-1}$ after UVB irradiation. Also, the peak area of proteoglycan region $(1,000$ $1,100 \mathrm{~cm}^{-1}$ ) significantly increased with the in- 


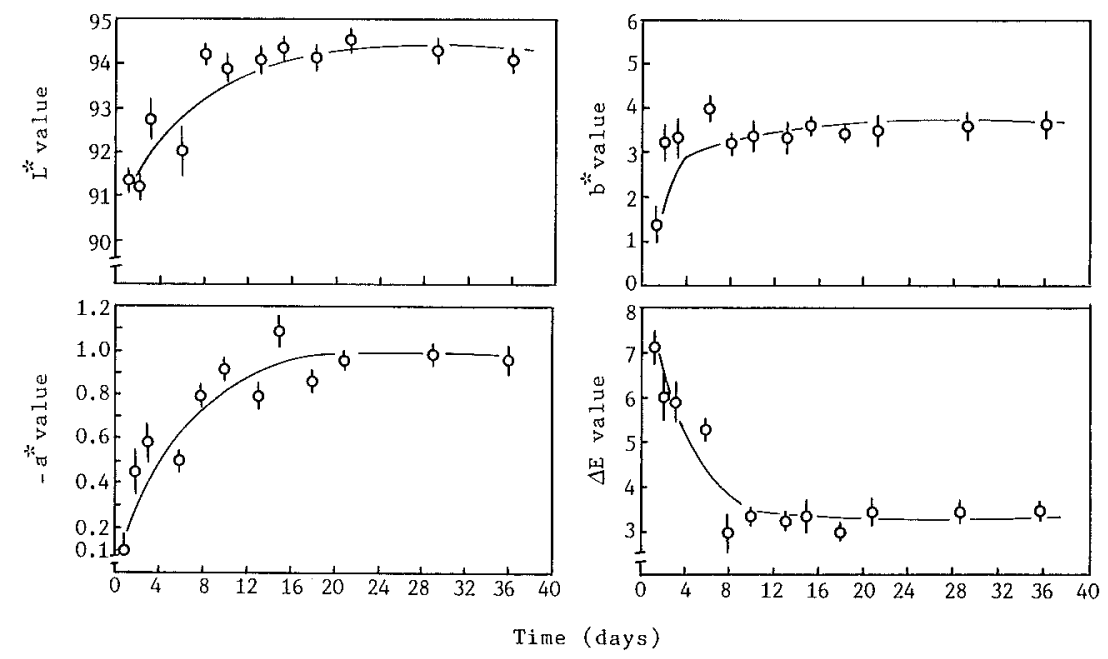

Fig. 1 Time-response curves of chromaticity $a^{*}, b^{*}$ and $L^{*}$ coordinates as well as $\Delta \mathrm{E}$ color difference in the porcine stratum corneum. Vertical bars represent the standard deviations of measurements on three application sites.

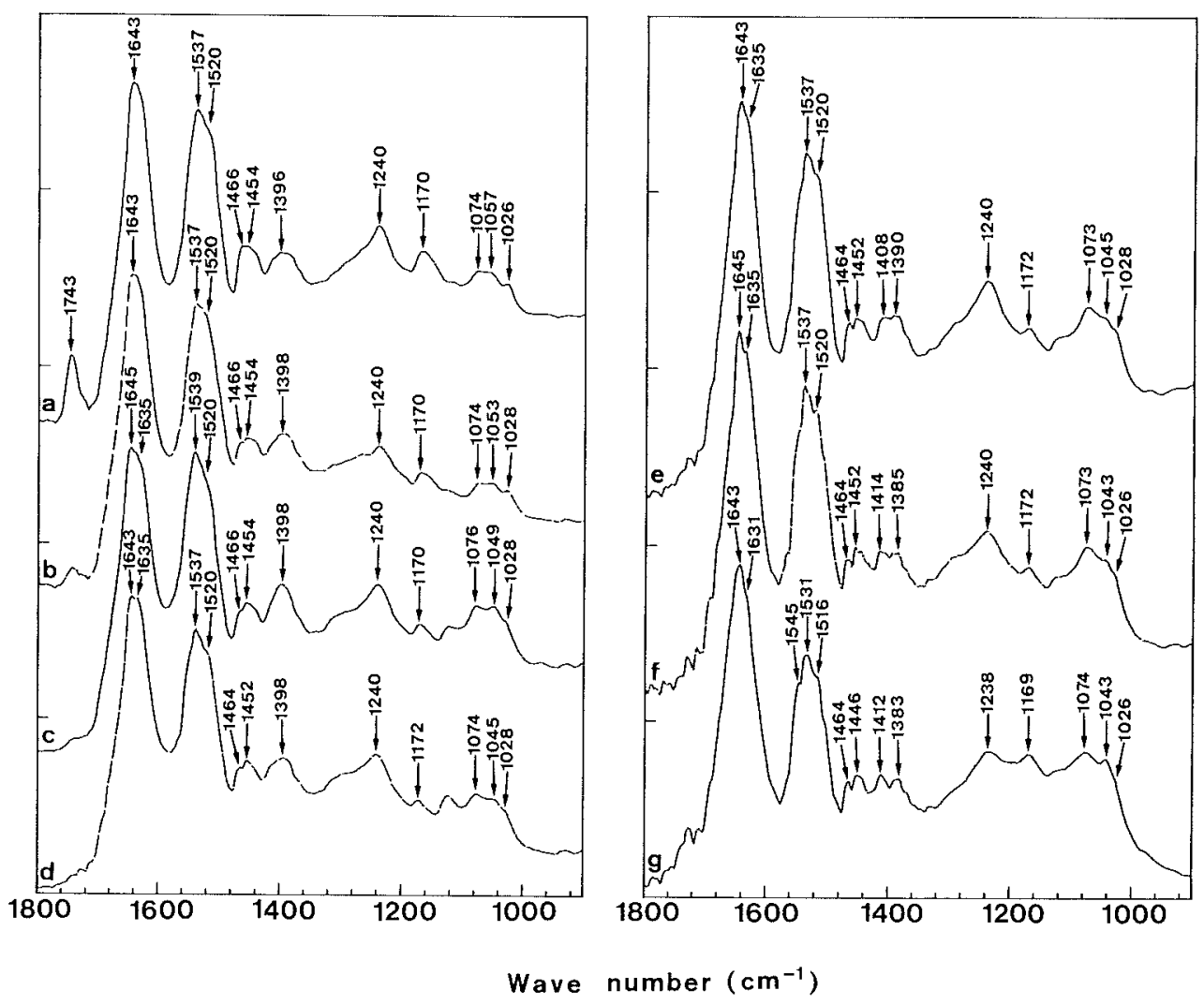

Fig. 2 Changes in IR spectra of porcine stratum corneum following UVB irradiation. (a) Porcine stratum corneum before UVB irradiation. (b-g) Porcine stratum corneum after UVB irradiation: (b) $16 \mathrm{~h}$; (c) $24 \mathrm{~h}$; (d) 2 days; (e) 10 days; (f) 18 days; (g) 36 days 


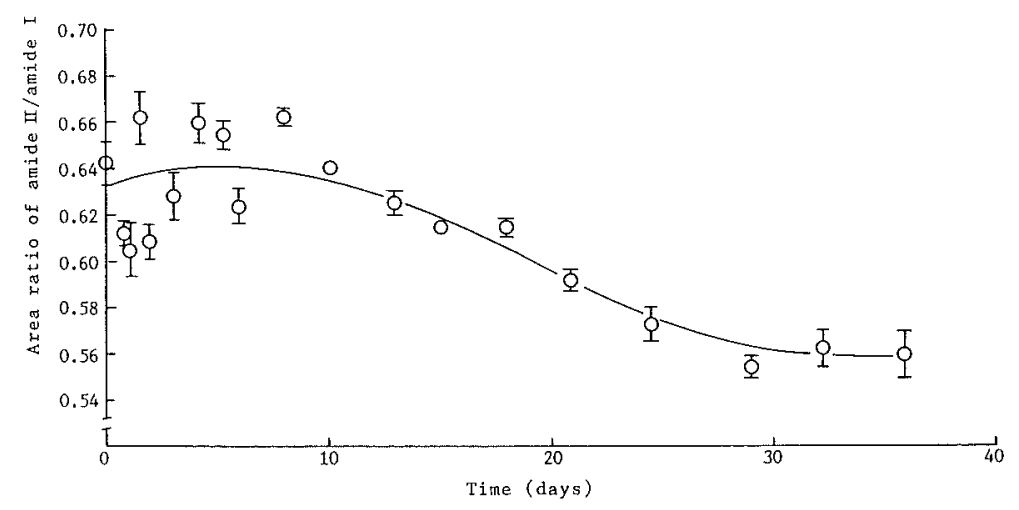

Fig. 3 Change in the peak area ratio of amide II/amide I of porcine stratum corneum after UVB irradiation

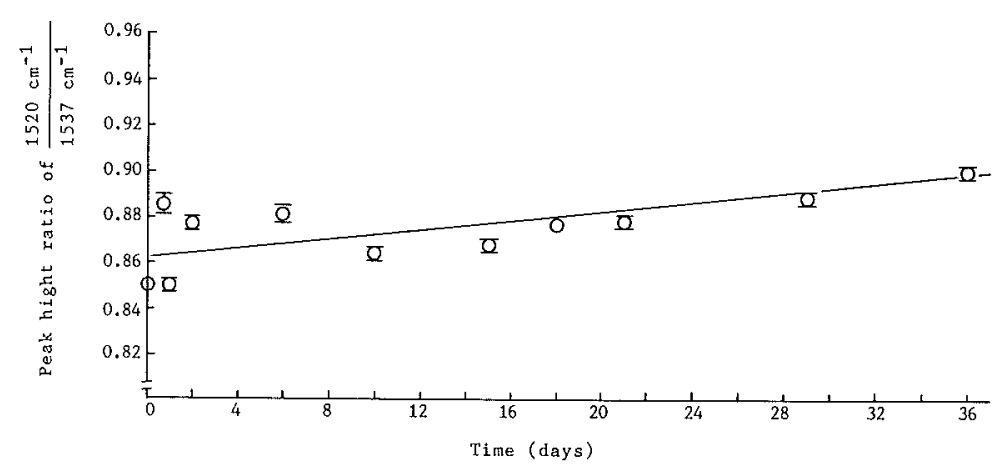

Fig. 4 Transition of $\alpha$-helix structure to $\beta$-sheet structure of porcine stratum corneum after UVB irradiation

crease in exposure time, which was consistent with the histological result of skin after UV irradiation (28).

Fig. 3 shows the peak area ratio of amide II to amide I. Obviously, the value of peak area ratio was almost constant for porcine SC following UVB irradiation for 10 days. However, this value decreased afterward due to the reduced peak area of amide II. On the other hand, the value of peak intensity ratio of $1,520 \mathrm{~cm}^{-1}$ to $1,537 \mathrm{~cm}^{-1}$ increased with the exposure time (Fig. 4). The secondary structure of $\alpha$-helix that was transformed into $\beta$-sheet structure after UVB exposure seems to be responsible for this result.

\section{DISCUSSION}

In the present study we investigated the timedependent changes in the secondary conformational structure of isolated porcine SC after UVB irradiation. Since UV irradiation is absorbed or scatters mostly in the upper epidermis, the damage therein is apparently more serious than in the lower epidermal cells. ATR measurement, consisting of FT-IR spectrometer equipped with an MCT detector and a zinc selenide ATR prism, is suitable for detecting the secondary conformational structure of the upper epidermal layer, whose thickness is only $0.1-2 \mu \mathrm{m}$.

Skin color after UV irradiation depends on multiple factors, the most important of which are erythema, tanning and melanin pigmentation. Melanin explains the erythroma and tanning. In the living skin, melanin pigments serve to protect the inner skin from damage of light. Thus the skin following light exposure becomes slowly darkening, leading to an increase in the $A^{*}$ value and a decrease in the $L^{*}$ value. In the present study, however, with both colorimeters a negative movement on the $\mathrm{A}^{*}$-axis and a positive movement on the $L^{*}$-axis were obtained. Although the $L^{*}$ value of skin surface is a complex factor influenced not only 


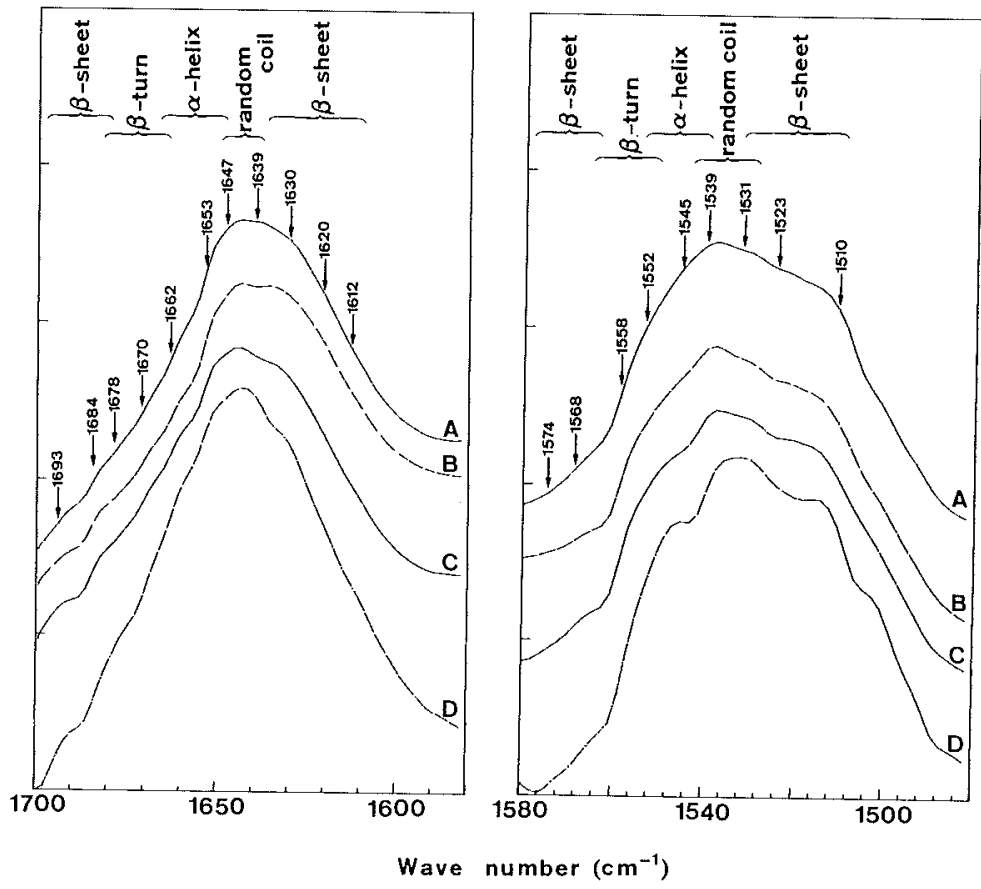

Fig. 5 IR absorption spectra of porcine stratum corneum in amide I and II regions. (A) Before UVB irradiation. (B-D) After UVB irradiation: (B) 2 days; (C) 10 days; (D) 36 days

by chromophores in the skin, but also by factors which change the skin surface reflectiveness like skin dryness or roughness, structural differences in the epidermis may also influence scattering, reflective light and the $L^{*}$ value $(2,24)$. The opposite results may be mainly attributable to the absence of pigment formation in the isolated porcine SC. Other factors such as skin dryness or roughness and surface structural differences might also contribute to the opposite results.

Some reports indicate that total lipids of healthy human SC increase after UV irradiation to enhance intercelluar cohesion to hold horny cells together $(5,15)$. In our study, however, IR peaks at 1,743 and $1,466 \mathrm{~cm}^{-1}$, assigned to the carbonyl and scissoring bands of lipid structure, disappeared with the increase of exposure time, suggesting that lipid was reduced following UVB irradiation, possibly because the $\mathrm{SC}$ was isolated from the living condition. The position and assignments of amide I and amide II of porcine SC after UVB irradiation are shown in Fig. 5. The estimated compositions of secondary structure were obtained from IR absorptions for the characteristic amide I and II bands: $\alpha$ helical structure at 1,650-1,660 and 1,550-1,537 $\mathrm{cm}^{-1}, \beta$-sheet structure at $1,625-1,635,1,670-$
1,685 and $1,510-1,530 \mathrm{~cm}^{-1}, \beta$-turn structure at $1,665-1,670,1,685-1,695$ and $1,530-1,565 \mathrm{~cm}^{-1}$, and random coil structure at 1,640-1,645 and $1,530-1,540 \mathrm{~cm}^{-1}(11,29)$. The secondary structure of protein in porcine SC changed with the increase in exposure time. The amide I peak at $1,635 \mathrm{~cm}^{-1}$ appeared clearly after one-day exposure (Figs. $2 \mathrm{c}$ and 5B), but it shifted to $1,631 \mathrm{~cm}^{-1}$ following exposure for 36 days. The peaks for amide II exhibited more prominent transformation than did amide I peaks. After 36-day exposure, the amide II peak split into three peaks at 1,545, 1,531 and $1,516 \mathrm{~cm}^{-1}$. The peak at $1,545 \mathrm{~cm}^{-1}$ was obviously found on the 36th day of exposure. However, the peaks at 1,531 and $1,516 \mathrm{~cm}^{-1}$ were derived from 1,537 and 1,520 $\mathrm{cm}^{-1}$, respectively (Figs. $2 \mathrm{~g}$ and $5 \mathrm{C})$. The transformation of the secondary structure of porcine SC after UVB irradiation is also shown in Fig. 4. The peak at $1,537 \mathrm{~cm}^{-1}$ assigned to $\alpha$ helix structure was transformed into $\beta$-sheet structure at $1,520 \mathrm{~cm}^{-1}$. Fig. 3 also indicates that the peak area of amide II is lowered with the increase in exposure time. The result is consistent with our previous observation, in which the collagen was denatured by compression and the peak area of amide II significantly decreased (16). This implies 
that the protein in porcine SC was perhaps partially denatured after UVB irradiation, leading to a decrease in the peak area of amide II. The peak at $1,454 \mathrm{~cm}^{-1}$ assigned to the $\mathrm{CH}_{3}$ asymmetric bending of protein shifted to $1,446 \mathrm{~cm}^{-1}$ after UVB exposure. The peak at $1,396(1,398) \mathrm{cm}^{-1}$, assigned to $\mathrm{CH}_{2}$ and $\mathrm{CH}_{3}$ deformation and/or $\mathrm{COO}^{-}$symmetric stretching band, split into two peaks at 1,412 and $1,383 \mathrm{~cm}^{-1}$, respectively, assigned to the scissoring vibration of alkenes, which implies that, the alkane bonds of protein in the porcine $\mathrm{SC}$ became unsaturable after UVB irradiation $(19,23)$. It has been reported that $\mathrm{C}-\mathrm{O}$ absorption shifts to lower frequency when unsaturation is present on adjacent carbon (23). The present observation that the peak at $1,057 \mathrm{~cm}^{-1}$ assigned to the $\mathrm{C}-\mathrm{O}$ bending and stretching bands of glycogen shifts to $1,043 \mathrm{~cm}^{-1}$ after UVB irradiation, implies that the unsaturation of glycogen occurs after UVB irradiation.

Since the major effect of UV irradiation is the increasing production of free radicals, during the latent period of UV irradiation, generated free radicals can attack, infiltrate and injure epidermis and dermis, resulting in an increase in conjugated dienes and lipid peroxidation products (8). This conclusion is consistent with our present findings that UVB irradiation can unsaturate the protein and lipid of the isolated skin. How the changes in secondary structure affect the living skin after photo-damage remains to be known.

The authors thank the Chin-Lin Medical Foundation for their financial support (CI 81-27).

Received 24 September 1993; and accepted 6 December 1993

\section{REFERENCES}

1. Adhoute H., de Rigal J., Marchand J. P., Privat Y. and LEVEQUE J. L. (1992) Influence of age and sun exposure on the biophysical properties of the human skin: an in vivo study. Photodermatol. Photoimmunol. Ph. 9, 99-103

2. AGNER T. (1991) Basal transepidermal water loss, skin thickness, skin blood flow and skin colour in relation to sodium-lauryl-sulphate-induced irritation in normal skin. Contact Dermatitis 25, 108-114

3. Andersen P. H., Abrams K. and Maibach H. (1992) Ultraviolet $B$ dose-dependent inflammation in humans: a reflectance spectroscopic and laser Doppler flowmetric study using topical pharmacologic antagonists on irradiated skin. Photodermatol. Photoimmunol. Ph. 9, 17-23

4. Carpenter J. F. and Crowe J. H. (1989) An infrared spectroscopic study of the interactions of carbohydrates with dried proteins. Biochemistry 28, 3916-3922
5. Chatenay F., Corcuff P., Saint-Leger D. and Leveque J. L. (1990) Alterations in the composition of human stratum corneum lipids induced by inflammation. Photodermatol. Photoimmunol. Ph. 7, 119-122

6. Danno K., Horio T. and Imamura S. (1992) Infrared radiation suppresses ultraviolet B-induced sun-cell formation. Arch Dermatol. Res. 284, 92-94

7. Forbes P. D., Blum H. F. and Davies R. E. (1981) Photocarcinogenesis in hairless mice: dose-response and the influence of dose-delivery. Photochem. Photobiol. 34, 361-365

8. Fuchs J, and PACKer L. (1990) Ultraviolet irradiation and the skin antioxidant system. Photodermatol. Photoimmunol. Ph. 7, 90-92

9. Goodman M. and Barry B. W. (1986) Differential scanning calorimetry of human stratum corneum: Effects of penetration enhancers azone and dimethyl sulphoxide. Anal. Proc. 23, 397-398

10. Haris P. I. and Chapman D. (1992) Does Fourier-transform infrared spectroscopy provide useful information on protein structures? Trends Biochem. Sci. 17, 328-333

11. Havel H. A., Chao R. S., Haskell R. J. and Thamann T. J. (1989) Investigations of protein structure with optical spectroscopy: Bovine growth hormone. Anal. Chem. 61, 642650

12. Khan Z. U. and Kellaway I. W. (1989) Differential scanning calorimetry of dimethylsulphoxide-treated human stratum corneum. Int. J. Pharm. 55, 129-134

13. Kligman L. H., Akin F. J. and Kligman A. M. (1985) The contributions of UVA and UVB to connective tissue damage in hairless mice. J. Invest. Dermatol. 84, 272-276

14. Knutson K., Krill S. L., LAmbert W. J. and Higuchi W. I. (1987) Physicochemical aspects of transdermal permeation. J. Control. Release 6, 59-74

15. Lehmann P., Hölzle E., Melnik B. and Plewig G. (1991) Effects of ultraviolet $\mathrm{A}$ and $\mathrm{B}$ on the skin barrier: a functional, electron microscopic and lipid biochemical study. Photodermatol. Photoimmunol. Ph. 8, 129-134

16. Lin S.-Y., Hou S.-J., Hsu T. H.-S. and Yer F.-L. (1992) Comparisons of different animal skins with human skin in drug percutaneous penetration studies. Meth. Find. Exp. Clin. Pharmacol. 14, 645-654

17. LiN S.-Y. and Lin T.-C. (1993) Repairable function of pyrrolidone-carboxylate sodium to restore the disordered skin induced by enhancers. Proc. Intern. Symp. Control. Rel. Bioact. Mater: 20, 436-437

18. Mantsch H. H., Yang P. W. and CASAL H. L. (1986) Infrared spectrometry of living systems: Current trends and perspectives. J. Mol. Struct. 141, 237-242

19. Morrison R. T. and Boyd R. N. (1987) Organic Chemistry, Allyn and Bacon, Boston, pp. 249-295

20. Muramatsu T., Kobayashi N., Tada H., Yamaji M., ShIRAI T., MORI T, and OHNISHI T. (1992) Induction and repair of UVB-induced cyclobutane pyrimidine dimers and (6-4) photoproducts in organ-cultured normal human skin. Arch. Dermatol. Res. 284, 232-237

21. Oertel R. P. (1977) Protein conformational changes induced in human stratum corneum by organic sulfoxides: An infrared spectroscopic investigation. Biopolymers 16, 2329-2345

22. OIKARINEN A. (1990) The aging of skin: chronoaging versus photoaging. Photodermatol. Photoimmunol. Ph. 7, 3-4

23. Pavia D. L., Lampman G. M. and Kriz G. S. (1979) Introduction to Spectroscopy: A Guide for Students of Organic Chemistry. W. B. Saunders, Philadelphia, pp. 13-80 
24. Rox Anderson R. and Parrish J. A. (1981) The optics of human skin. J. Invest. Dermatol. 77, 13-19

25. SAYRE R. M. (1992) Action spectra: Sunlight and you. Cosmet. Toil. 107, 59-62

26. Schwartz E., Cruickshank F. A., Perlish J. S. and Fleischmajer R. (1989) Alterations in dermal collagen in ultraviolet irradiated hairless mice. J. Imvest. Dermatol. 93, 142-146

27. Seitz J. C. and Whitmore C. G. (1988) Measurement of erythema and tanning responses in human skin using a tristimulus colorimeter. Dermatologica 177, 70-75

28. Smith J. G., Davidson E. A., Tindall J. P. and Sams W. M.
(1961) Hexosamine and hydroxyproline alterations in chronically sun-damaged skin. Proc. Soc. Exp. Biol. Med. 108, 533-535

29. Wantyghem J., Baron M.-H., Picquart M. and Lavialle F. (1990) Conformational changes of Robinia pseudoacacia lectin related to modifications of the environment: FTIR investigation. Biochemistry 29, 6600-6609

30. Westerhof W., Estevez-Uscanga O., Meens J., Kammeyer A., Duroce M. and Cario I. (1990) The relation between constitutional skin color and photosensitivity estimated from UV-induced erthema and pigmentation doseresponse curves. J. Invest. Dermatol. 94, 812-816 